\title{
Non-accidental injury in companion animals in the Republic of Ireland
}

\author{
Kristina McGuinness', Mary Allen² and Boyd R. Jones' \\ ' Faculty of Veterinary Medicine, University College Dublin, Dublin 4. \\ ${ }^{2}$ Department of Social Policy and Social Work, University College Dublin, Dublin 4.
}

Non-accidental injury (NAI), animal abuse and "battered pet" syndrome are terms used to identify "the intentional harm of an animal". The terms include, but are not limited to, wilful neglect, inflicting injury, pain or distress, or malicious killing of an animal. Three categories of abuse are recognised: physical, sexual and neglect.

A postal survey was conducted to determine the extent to which NAI was recognised by veterinary surgeons in urban, semi-rural and rural veterinary practices in the Republic of Ireland. The questionnaire was sent to 600 veterinarians; completed submissions were received from I I 5 respondents (19.2\%).

The occurrence of NAI was acknowledged by 106 (92.2\%) of the respondents and cases had been seen by 50 (43.3\%) of them, comprised of $36.2 \%$ of urban veterinary surgeons from rural towns and of $82 \%$ of urban practitioners. In 59\% of cases the client indicated the injury was non-accidental; 39 (67.2\%) of the 58 reported cases involved a single event. Signs that made veterinary surgeons suspicious of NAl included inconsistent history, untreated injuries, recurring injuries, meekness of the animal, suspicious behaviour of the owner and injuries consistent with abuse. The types of injuries observed included burns, lacerations, gunshot wounds, poisoning, injury to genitalia, bruising and fractures.

The findings of this study are comparable with those from other countries. Most but not all veterinary surgeons in Ireland recognise NAl and animal abuse is of significant concern in rural and urban communities as evidenced by this survey of practising veterinary surgeons.

\section{Introduction}

Non-accidental injury (NAI), 'animal abuse' or 'battered pet syndrome', is a phenomenon increasingly recognised by veterinary surgeons in practice. These terms have different interpretations and meanings in different societies and an absolute definition has been difficult to determine (Munro and Thrusfield, 200 la). The National Society for the Prevention of Cruelty to Children (NSPCC 2003: Weston House, 42 Curtain Road, London EC2A 3NH, England) has described animal abuse as "the intentional harm of an animal". It includes, but is not limited to, wilful neglect, inflicting injury, pain or distress, or malicious killing of animals. In light of recent research, the organisation has defined three categories of animal abuse: physical abuse, sexual abuse and neglect. Physical abuse includes kicking, punching, throwing, burning, microwaving, asphyxiation, and the administration of drugs or poisons. Sexual abuse encompasses the use of an animal for sexual gratification. Neglect is a failure to provide adequate food, water, shelter, companionship or veterinary attention.

\section{Corresponding author:}

Boyd Jones Faculty of Veterinary Medicine,

University College Dublin, Dublin 4, Ireland

Tel: +353 । 7166103

Fax:+353।7166104

E-mail:boyd.jones@ucd.ie
Guidelines published by the Professional Conduct Department of the Royal College of Veterinary Surgeons in the United Kingdom (UK) state: "when a veterinary surgeon is presented with an injured animal whose clinical signs cannot be attributed to the history provided by the client, s/he should include non-accidental injury in the differential diagnosis".

A study carried out by Munro and Thrusfield (200la,b,c,d) in the United Kingdom, investigating the experiences of I,000 small animal veterinary practitioners, highlighted the alarming levels of NAI encountered by veterinary practitioners on a daily basis. This groundbreaking work established basic guidelines for the recognition of NAl in dogs and cats and highlighted just how extensive and pervasive a problem existed. However, to date a similar investigation had not been carried out in Ireland. Information gathered in the UK study indicated that the profession embodied a large amount of knowledge on the subject of NAl that had not been recorded or shared. It is likely that veterinary surgeons in Ireland are witnessing evidence of animal abuse on a daily basis, in many cases possibly without realising it, and possibly witnessing evidence of domestic abuse also. The link between animal abuse and domestic abuse has been well established (DeViney et al., 1983;Arkow, 1994).

A seminar, hosted by the Faculty of Veterinary Medicine, University College Dublin (UCD) in January 2003, highlighted how little is known regarding the extent of NAI in Ireland (Boyle, 2003). Given the longrunning speculation and research into this area in other societies, 
TABLE I: Summary of the types, the causes and the locations of injuries in the 58 animals

\section{Type of injury}

Gunshot wound

Pellet wound

Burn 4

Bruising 2

Laceration 3

Stab wound 3

Multiple fractures

Skull fractures

Depressed rib fractures

Poisoning

Injury to genitalia

\section{Location of injuries}

Limbs

Thorax

Other

2

$3 \quad 6$

22

$5 \quad 4 \quad$ I

I 4

2

4 and the noted lack of similar studies in Ireland, it was appropriate to investigate the extent and circumstances surrounding NAI in companion animals as seen by veterinary practitioners in this country. The aims of this study were three-fold: to determine the extent to which veterinary surgeons suspect occurrences of NAl in small animals in Ireland; to determine the features by which veterinary practitioners in Ireland recognise cases of NAl; and to investigate the potential link between NAI in companion animals and domestic violence in this country.

\section{Materials and methods}

A sampling frame was constructed from the list of members of Veterinary Ireland resident in the Republic of Ireland. The survey was posted to 299 companion animal practitioners, which represented the entire membership of declared companion animal practitioners, and to $30 \mathrm{I}$ mixed practice practitioners from the total of $600 \mathrm{food}$ animal and/or equine practitioners selected randomly from the Veterinary Ireland database.

The questions asked were based on the study of Munro and Thrusfield (200la). Accompanying the submission forms was a cover page explaining the purpose of the survey. The survey itself consisted of a set of general questions about the person filling in the survey and three case report sheets. There was also a final section for free text commentary and a freepost envelope to return the survey.

General questions about the person completing the questionnaire were: gender of the respondent, age of the respondent, length of time in veterinary practice, if $s /$ he practised in a rural or urban area and in either a small animal practice or a mixed practice (and, if mixed, approximately what percentage of the practice was devoted to small animals). The respondent was asked if s/he was aware that NAI occurs to small animals and, finally, if s/he had ever suspected or seen NAI to animals in the practice.

The case report sheets required completion of details of cases of $\mathrm{NAI}$, including the approximate date of occurrence, species, breed, sex and age of the animal involved. A question asked whether the client had stated the injury was non-accidental. Another question enquired who had caused the injury, if that had been recorded. The respondent was asked to specify what had aroused a suspicion that the injury was non-accidental. If the case involved single or multiple incidents, the respondent was asked to give a detailed account of injuries seen and the outcome of the case. Finally, the respondent was asked if $s /$ he felt that the NAI was part of a wider abuse within the family and, if so, why? Respondents were also asked what they felt were their options for assisting the animal and client and if they would feel comfortable displaying information about domestic violence in their waiting rooms for clients to view.

Data analysis

The information recorded on the questionnaires was labelled numerically and transferred to a custom-built database using Microsoft Access 2000. Responses to open questions were entered in abbreviated form. The data were analysed using the statistical package SPSS 8.0 .

\section{Results}

Response

Over a three-month period, completed questionnaires were returned by 115 respondents (19.2\%), of whom 61 practised in rural areas (57 in mixed practices and four in small animal practices), 53 respondents worked in urban-based practices (23 in mixed practices and 30 in small animal practices), and one respondent did not answer that question.

\section{Incidence of NAI}

Cases of NAl had been seen by 65 respondents, comprising 22 (36.2\%) of the 62 rural practitioners and $43(82.0 \%)$ of the 53 urban practitioners, while $106(92.2 \%)$ of the respondents were aware that $\mathrm{NAI}$ occurs in companion animals.

Fifty respondents recorded 58 cases of NAI (Figure I). These cases involved 53 dogs, four cats, and one rabbit. Sixteen cases (30.1\%) in dogs involved collies or collie cross dogs and eight cases (I5.I\%) involved crossbred dogs. All reported incidences in cats involved domestic short hair cats. Animals of all ages were represented in the case reports (Figure 2).

In 35 of the cases $(60 \%)$ the client had indicated that the injury was non-accidental. According to the clients, injuries had been inflicted by a neighbour (22 cases), a partner/boyfriend/girlfriend (five cases), the owner's spouse (one case), the client (three cases), a relative (three 


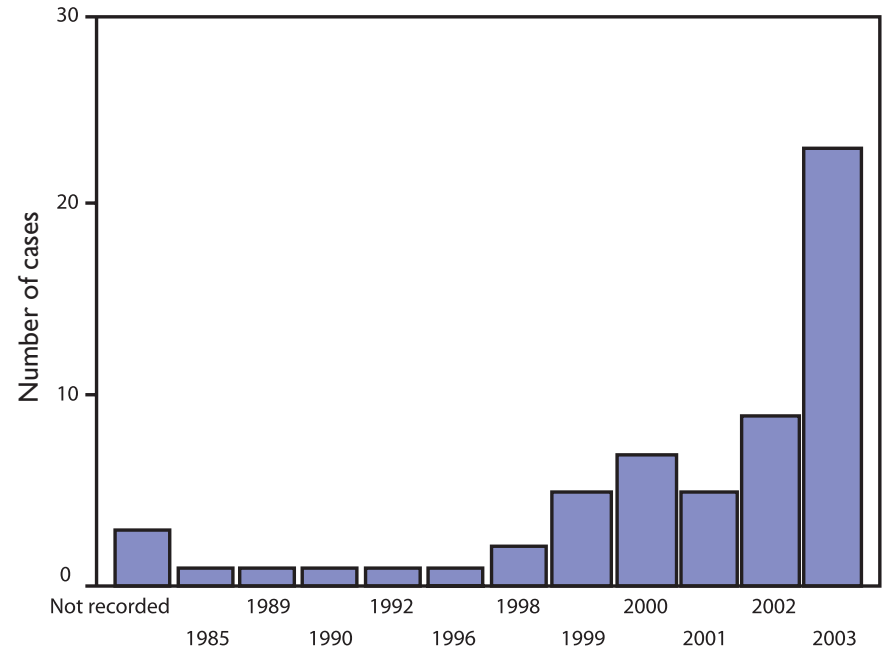

FIGURE I: Fifty-eight cases of non-accidental injury: displayed by year of occurrence.

cases) or a colleague (one case).

\section{Reported injuries}

Injuries reported (Table I) included: burns to the head and neck, thorax, abdomen, limbs and dorsum; lacerations to the head, abdomen and limbs; stab wounds to the head, neck, thorax, abdomen and dorsum; gunshot wounds to the thorax and abdomen; pellet wounds to the limbs; bruising; non-accidental multiple limb fractures; depressed rib fractures; skull fractures; poisoning and injury to genitalia.

Two-thirds of the reported cases involved a single event: however, in one-third of the 'single incident' cases the animal either died or was euthanased because of the injuries (Table 2).

Signs that made veterinary practitioners suspect that injuries were non-accidental were: injury that was inconsistent with history; old untreated fractures; meekness of the animal or an excessive fear of strangers or of men. Other suspicious signs were recurring injuries, injuries that seemed consistent with abuse (e.g., head trauma, string tied to genitals, gunshot or pellet wounds, stab wounds, cigarette burns) and the suspicious behavior of the owner/client. In some cases a history of violence within the family aroused suspicion when the pets of these families presented with unusual traumatic injuries, as did a high turnover of pets within a household (Table 3).

Further comments

Forty-eight (42\%) of responding veterinary surgeons made further comments in the free text section. One practitioner stated that guidelines for the recognition and assessment of cases would be helpful.Three respondents wrote that they were previously

TABLE2:The outcome of the 58 cases of non-accidental injury

\begin{tabular}{lcl} 
Outcome & Number of animals & Per cent \\
Died & 12 & 20.7 \\
\hline Euthanased & 6 & 10.3 \\
\hline Survived & 31 & 53.4 \\
\hline Unknown & 9 & 15.5
\end{tabular}

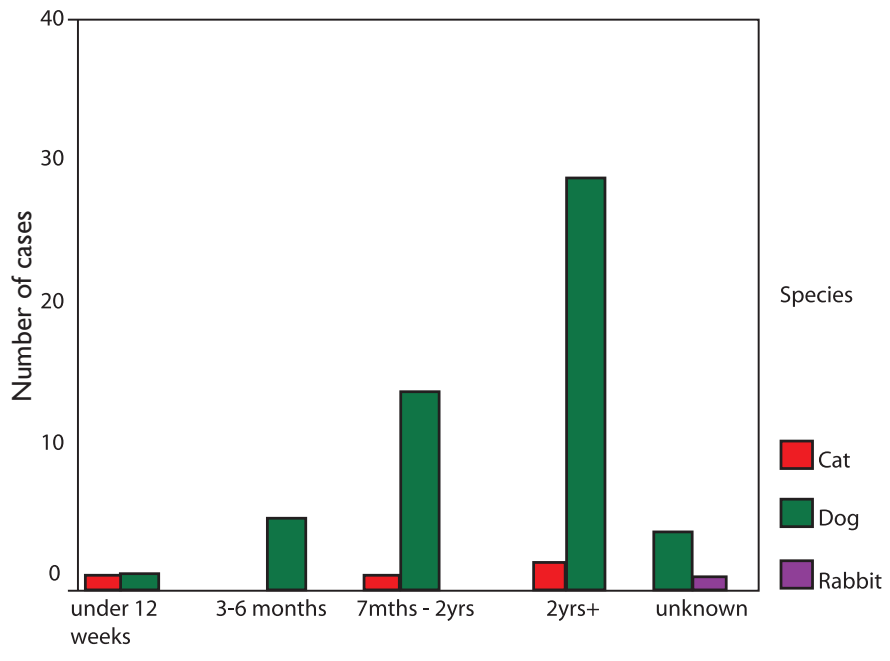

FIGURE 2: Age distribution of the 58 reported cases of non-accidental injury according to species.

unaware that NAI cases were something that they could be seeing in practice and, in hindsight, they felt that cases of abuse may have been presented but not identified because they were not aware of the significant signs. Another comment (two respondents) was that legislation dealing with this issue is inadequate and that suitable legislation should be in place, as appropriate legal assistance is required to deal with these cases. Three respondents stated that a similar study should be carried out regarding NAl as seen by large animal practitioners. One veterinary practitioner detailed a case involving sexual abuse of a dairy cow, and another respondent reported witnessing cases of NAl in farm animals "on a weekly basis".

\section{Response of veterinary surgeons to cases of $\mathrm{NAl}$}

Thirty-four (58.6\%) of respondents_did not answer the question about they dealt with the issue of NAI when presented with suspect cases. Of those who did answer the question the question, seven (I2.1\%) of veterinary surgeons felt that they had no option to assist the client; I4 ( 24. I\%) had contacted the Gardaí or local Society for Prevention of Cruelty to Animals (SPCA); three (5.2\%) referred the client to an appropriate support group/ relevant agency. In eight of the cases the

TABLE 3: Features that aroused suspicion of non-accidental injury in 58 animals

\section{Feature}

Number of

occasions recorded

Injuries that seemed consistent with abuse 12

Recurring injuries

Old untreated fractures

A history inconsistent with the injury

Animal showed excessive fear 3

Meekness of the animal

Suspicious behaviour of the client

।

High pet turnover 
attending veterinary surgeon suspected that the injury to the animal was part of a wider spectrum of abuse within the home.

\section{Discussion}

Due to the difficulty in obtaining a register of veterinary practices and addresses in Ireland, it was not possible to develop an unbiased sampling frame for the study. As a result of selection of the sampling frame from the database of Veterinary Ireland members, there is an element of bias in the results of this study. This bias, coupled with the low response rate of $19.2 \%$, means that caution should be exercised in interpretation of the results of the study, as the external validity is influenced by these factors. There is perhaps also a measurement bias in this study as the accuracy of the information obtained from the case reports is influenced by the accuracy of the memory of the respondent. In some instances case reports dated back as far as 1986. Arguably, the accuracy of human memory decreases over time and this has an influence on the internal validity of the results.

On the basis of these results it can be concluded that NAI in companion animals is a problem encountered by veterinary practitioners in Ireland, with $92.2 \%$ of the survey respondents acknowledging the existence of NAl in companion animals and $44.3 \%$ having seen cases of NAl in practice. These findings are remarkably similar to those of Munro and Thrusfield (200lb), in the UK, where $91.3 \%$ of the respondents acknowledged the existence of $\mathrm{NAl}$ in companion animals and $48.3 \%$ had seen cases of NAI in practice. In addition, similar types of injury were seen and many of the same warning signs were identified by survey groups in both countries.

As a result of the UK study, the RCVS published a set of guidelines for practising veterinarians outlining how they should react when faced with a case of NAI. In addition, two further booklets providing advice to the veterinary profession were published. One, entitled "Understanding the links; child abuse, animal abuse and domestic violence", is published by the National Society for the Prevention of Cruelty to Children (NSPCC 2003: Weston House, 42 Curtain Road, London EC2A 3NH, England) in association with the British Small Animal Veterinary Association, Blue Cross, Scottish Society for the Prevention of Cruelty to Animals, Royal Society for the Prevention of Cruelty to Animals, Peoples Dispensary for Sick Animals and various other organizations. This booklet provides guidelines regarding the action professionals should take if they have concerns about the abuse of children or animals. A second leaflet is aimed at providing veterinarians with guidelines on how to recognise animal abuse in their practice, and is entitled "Forging the Link" (Intervet UK, Walton Manor,Walton, Milton Keynes, MK7 7AY, England).

It is apparent from the results of this study that such guidelines would be beneficial to the veterinary profession in Ireland, to enable veterinary surgeons to recognise and respond appropriately to NAI cases.

In our study, it was a highly significant finding that in approximately one in eight cases of NAl, the treating veterinary surgeon was suspicious of further violence within the family. It raises difficult questions regarding the social obligations of veterinary practitioners to act on behalf of their patients and clients. The importance of the link between child abuse, animal abuse and domestic violence has been highlighted (Arkow, 200I). Research from the United States (De Viney et al., 1983) demonstrated that animal abuse had occurred in $88 \%$ of families with concurrent physical child abuse. In an UK study, $82 \%$ of families investigated by the RSPCA were known to social services agencies as having children at risk.
Veterinary surgeons have an obligation not only to the welfare of their patients but also to the welfare of their clients. It is apparent from the survey results that the majority of Irish veterinary surgeons surveyed do not feel it is appropriate or possible to become involved when faced with cases of NAl. The reasons for this reluctance have been well described by Arkow (200I). Many professionals are reluctant to get involved due to fear of litigation, fear that the client will be driven away, fear of erosion of the client base of the practice, fear that reporting will compromise the safety of the victim, lack of knowledge of available resources, a perception that no action will be taken, fear of physical retaliation by the perpetrator, a lack of widely-accepted standards of identification and inexperience in dealing with misleading histories. While these are all serious considerations and are difficult problems for veterinary surgeons, Arkow (1994) outlined compelling reasons for encouraging their involvement. Essentially, failure to get involved condones and perpetuates the violence, and also puts others at risk.

The RCVS guidelines for veterinary surgeons in the UK state that the treating veterinary surgeon should intervene in the manner outlined in the guidelines "if the veterinary surgeon considers on reasonable grounds that either the animals show signs of abuse or are at real and immediate risk of abuse - in effect where public interest in protecting an animal overrides the professional obligation to maintain client confidentiality". Arkow (1994) suggested that "the danger is to do nothing in the face of emerging empirical support that cruelty to animals and children are insidious, often intertwined manifestations of family violence. Veterinary practitioners are important participants in community health who may initiate positive social service interventions" and that ultimately these interventions will benefit.

One respondent stated that, in accordance with current Irish legislation, the Cruelty to Animals Act, 1997, "it is a criminal offence to abuse an animal and that although a veterinary surgeon has no power to remove an animal from its owner, the veterinary surgeon has the option of keeping the animal in for hospitalization and calling the Gardaí ". It seems there is a requirement for specific legislation to be implemented to provide veterinary surgeons with clearly defined legal guidelines, support, and the power to detain an animal if they have valid concern for its immediate safety.

The fact that many veterinary surgeons did not feel that they were able to recognize NAl cases indicates that, along with the publication of guidelines for practising veterinary surgeons, education needs to be provided to undergraduates to enable new graduates to recognize suspicious signs themselves and also help them to assist their colleagues. Given the close correlation of the findings of this study with the original work carried out Munro and Thrusfield (200I), it is our opinion that the guidelines published for veterinary surgeons and public health workers in the UK are applicable to NAI in Ireland. These guidelines would be of great assistance to veterinary surgeons in this country were they to be made available or circulated by the Veterinary Council or other relevant bodies.

A repeat study of a random sample of all veterinary practitioners in Ireland would increase the external validity of results and, as such, provide data more representative of the Irish veterinary population. Further study into the extent of NAl in large animals would also be of interest, as noted in the comments of two respondents who had encountered $\mathrm{NAl}$ in cattle. 


\section{Acknowledgements}

The authors thank Peter Bishton of Veterinary Ireland, Xerox Ireland, Intervet Ireland, the Irish Veterinary Council, the Dublin Society for the Prevention of Cruelty to Animals, and all the respondents to the survey. We also thank Daniel Collins, Tracy Clegg and Simon More for their assistance with the statistical analysis.

\section{References}

Arkow, P. (1994). Child abuse, animal abuse and the veterinarian. Journal of the American Veterinary Medical Association 204: 1004- 1007.

Boyle, C. (200I). The battered-pet syndrome. Irish Veterinary Journal 56: $|4|-\mid 42$.

DeViney, E., Dickert, J. and Lockwood, R. (1983). The care of pets within child abusing families. International Journal for the Study of Animal Problems 4: 321-329.

Munro, H.M.C. and Thrusfield, M.V. (200la). Battered pets: Features that raise suspicion of non-accidental injury. Journal of Small Animal Practice 42: 218-226.

Munro, H.M.C. and Thrusfield, M.V. (200 lb). Battered pets: Non-accidental physical injuries found in dogs and cats. Journal of Small Animal Practice 42: 279-290.

Munro, H.M.C. and Thrusfield, M.V. (200/c). Battered pets: Sexual abuse. Journal of Small Animal Practice 42: 333-337.

Munro, H.M.C. and Thrusfield, M.V. (200Id). Battered pets: Munchausen syndrome by proxy. Journal of Small Animal Practice 42 : 385-389. 